\title{
Light outcoupling efficiency of top-emitting organic light-emitting diodes
}

\author{
L. H. Smith, ${ }^{\text {a) }}$ J. A. E. Wasey, and W. L. Barnes \\ School of Physics, University of Exeter, Exeter EX4 4QL, United Kingdom
}

(Received 5 November 2003; accepted 24 February 2004)

\begin{abstract}
We report results obtained from modeling the light outcoupling efficiency of top-emitting organic light-emitting diode (OLED) structures and compare them with results from conventional substrate-emitting structures. We investigate two types of emissive material, small molecule and conjugated polymers, and study three different cathode materials; aluminum, silver, and calcium. We show that top-emitting OLEDs may have outcoupling efficiencies comparable to their substrate-emitting counterparts, and that the choice of cathode material is critical to the optical performance of the device. (C) 2004 American Institute of Physics. [DOI: 10.1063/1.1712036]
\end{abstract}

Organic light emitting diodes (OLEDs) are being commercially exploited, owing to the many appealing features they possess, notably the ease with which they may be fabricated. Conventional OLEDs are substrate emitting, with light emitted typically through a glass substrate. For display applications active matrix driven pixels are required and a good way to accomplish this is to make top-emitting OLEDs, i.e., for the emission to take place through the cathode. This would allow OLEDs to be incorporated onto a silicon substrate, thus enabling easier integration of light emitting and control components. Top-emitting OLEDs have recently been shown to offer an efficient way to generate light. ${ }^{1-3}$

Most aspects of OLED performance that impinge on device efficiency have now been optimized, but there is still scope for improving light outcoupling. A schematic of the structure we consider is shown in Fig. 1(a) with the organic light-emitting layer sandwiched between a reflective anode and a composite metal/indium-tin-oxide (ITO) cathode. We model the conventional substrate-emitting OLED structure [Fig. 1(b)] for comparison.

In order to optimize devices it is important to study the details concerning the amount of power lost to various decay channels. The decay of excitons within the emissive organic layer may result in outcoupled light, in power lost to guided modes ${ }^{4}$ including surface plasmon-polariton (SPP) modes, ${ }^{5}$ or lost as heat to one of the electrodes. SPPs result from the coupling between the free charges at the surface of a metal and electromagnetic radiation. ${ }^{6}$ This interaction leads to longitudinal surface charge density fluctuations that propagate along the interface combined with an oscillating EM field that decays exponentially away from the metallic surface.

Here we investigate how the position of the emitters within the organic layer changes the strength of the coupling to different modes, and thus the optical efficiency of the device. To do this we make use of a specially adapted classical technique to calculate the power lost by an emissive dipole in a multilayered structure, ${ }^{7}$ with the dipole field being represented by a sum of plane waves. Each plane wave is characterized by a different in-plane wave vector, $k_{x}$, where $k_{x}$ is the component of the wave vector parallel to the interfaces. By calculating the power dissipated by the dipole as a

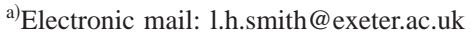

function of $k_{x}$ we are able to produce a power dissipation spectrum of the system, an example of which is shown in Fig. 2(a). Peaks in this spectrum indicate that power from the source is being lost to modes of the structure. To deduce the amount of power coupled to a specific mode, the area under the relevant peak in the spectrum is found by integration. By calculating the power dissipated as a function of both frequency and in-plane wave vector we can build up a dispersion diagram of the modes of the system as shown in Fig. 2(b). Dark features correspond to strong coupling between the emitter and the modes of the structure.

We studied three different cathode materials, namely Al, $\mathrm{Ag}$, and $\mathrm{Ca}$. Top-emitting structures and the corresponding substrate emitters were modeled for each type of cathode. We also investigated two types of emissive layer, the first was based upon the small molecule, aluminum tris-8hydroxyquinoline $\left(\mathrm{Alq}_{3}\right)$, the second on the conjugated polymer, poly[2-methoxy,5-(2'-ethyl-hexyloxy)-1,4-phenylenevinylene] (MEH-PPV). One of the main differences between these two materials is the fact that the dipole moments associated with the emitters have different orientations. Those in the $\mathrm{Alq}_{3}$ are assumed to have no preferred orientation, the dipole moment associated with the exciton is constantly diffusing, sampling all directions in space in a time less than the exciton lifetime. ${ }^{8}$ By contrast, in MEH-PPV the emitters lie predominantly in the plane of the layer due both to their long chain nature and the spin casting fabrication technique used. ${ }^{9}$ We will see that this difference is important in determining which modes the emitters are coupled to. The peak emission wavelength of $\mathrm{Alq}_{3}$ occurs at $550 \mathrm{~nm}$ and that of MEH-PPV at $580 \mathrm{~nm}$.

The top-emitting structures modeled here follow those
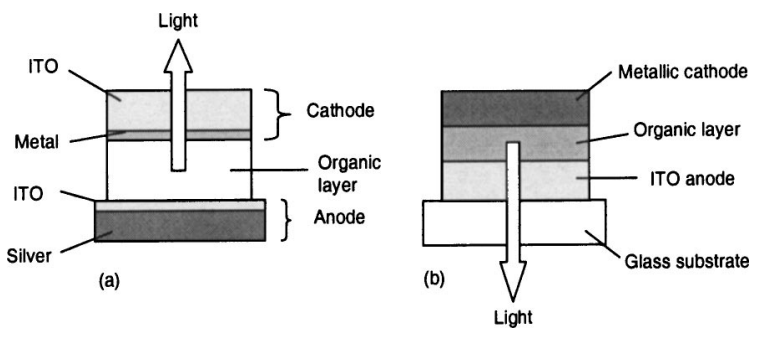

FIG. 1. (a) Generic top-emitting OLED, and (b) the generic substrateemitting OLED structures. 

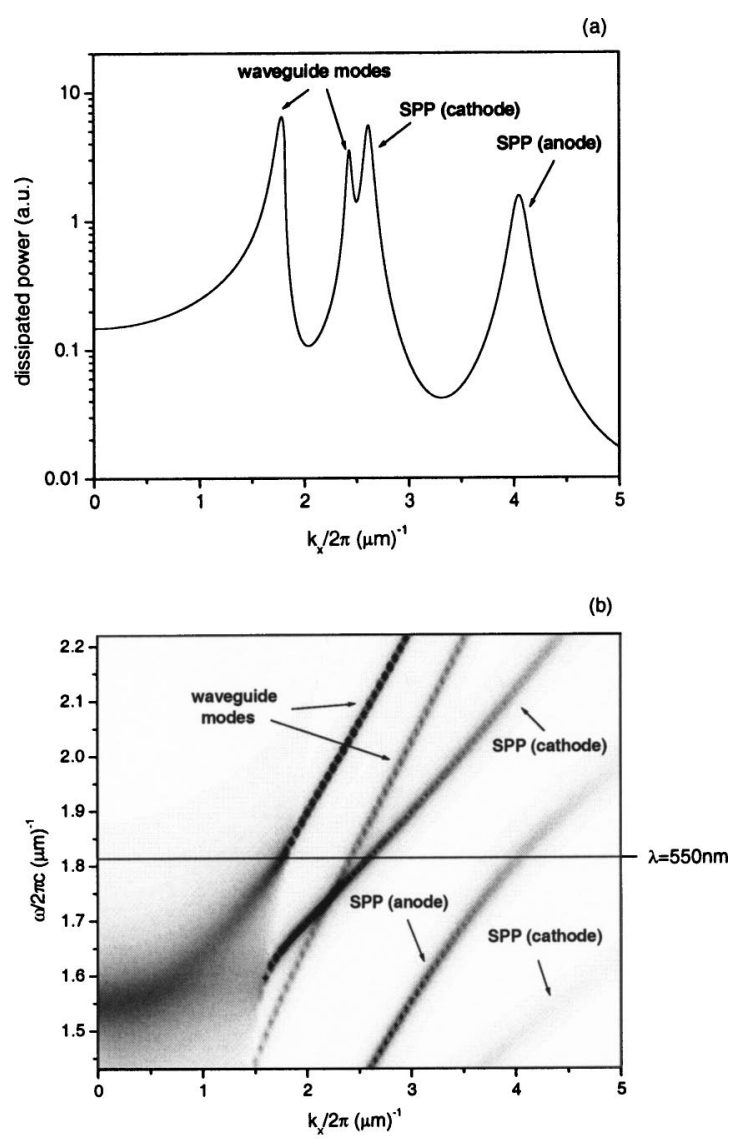

(c)

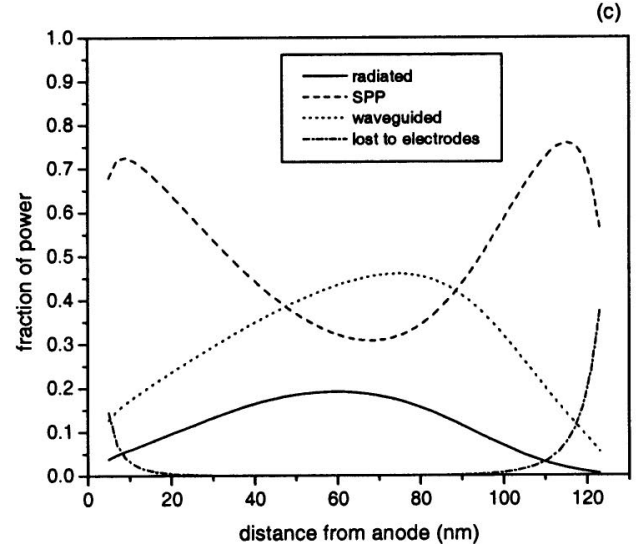

FIG. 2. Results for a top-emitting structure based upon the $\mathrm{Alq}_{3}$ emissive layer and a silver cathode. They show (a) a power dissipation plot for a single emission wavelength of $550 \mathrm{~nm}$, (b) a dispersion diagram, with the nature of the different modes indicated, and (c) a fraction of power plot for the structure.

studied by Lu et al., ${ }^{2}$ and consist of a reflective composite anode [Ag topped with $16 \mathrm{~nm}$ of indium tin oxide (ITO)], an organic layer $130 \mathrm{~nm}$ thick, and a cathode comprising $20 \mathrm{~nm}$ of the metal under investigation capped with $80 \mathrm{~nm}$ of ITO. The substrate-emitting structures consist of a thick, reflective metal cathode, a $130 \mathrm{~nm}$ organic emissive layer, a $130 \mathrm{~nm}$ layer of ITO (the anode), and a thick glass substrate through which the light is emitted. The refractive indices of the materials involved, their dispersion, and also the birefringence of the polymer were all incorporated into the model, with parameters taken from the literature. ${ }^{10,11}$

First we consider the $\mathrm{Alq}_{3}$ based system. In what follows, we plot the power lost to each decay channel as a Downloaded 25 Jul' 2008 to 144.173 .6 .75 . Redistribution subject

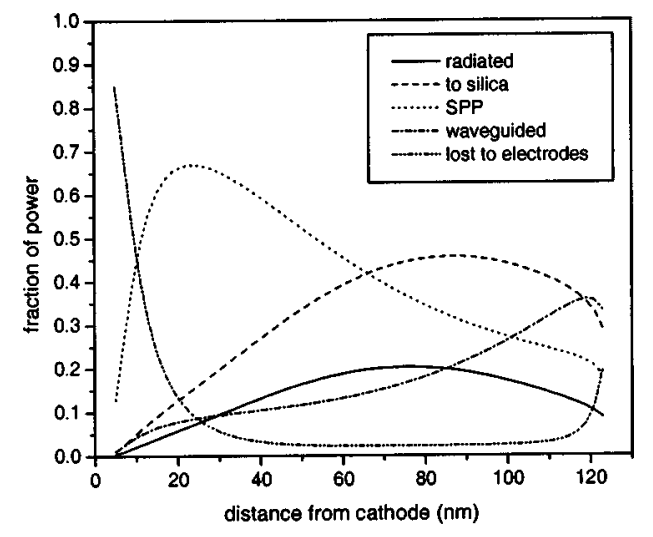

FIG. 3. Fraction of power plot for a substrate-emitting structure with an $\mathrm{Alq}_{3}$ emissive layer and a silver cathode.

fraction of the total lost to all channels (except nonradiative decay) as a function of the position of the emitters within the organic layer.

Such a plot for a top-emitting structure with a silver cathode is shown in Fig. 2(c). In contrast to substrate emitters, the presence of two metallic electrodes in the top emitters leads to the possibility of more complex SPP modes [Figs. 2(a) and 2(b)]. Indeed in the cases of Ag [Fig. 2(c)] and $\mathrm{Al}$ cathodes, we observed two peaks in the fraction of power lost to SPP modes, one occurring when the emitters are closer to the anode and the other when they are closer to the cathode. The maximum optical outcoupling efficiencies of the top-emitting $\mathrm{Ag}$-, $\mathrm{Al}-$, and $\mathrm{Ca}$-based structures are $19.2 \%, 6.6 \%$, and $16.6 \%$, respectively (with the emitters situated at their optimum location for light outcoupling). The low value for the $\mathrm{Al}$ cathode is in large part due to the skin depth of Al being significantly less than the cathode thickness.

Similar modeling for top-emitting OLED structures with a conjugated polymer (MEH-PPV) as the light-emitting material was also undertaken. For these structures the most significant power loss is found to be to the waveguide modes, with this contribution being at its highest when the emitters are located more centrally. Coupling to the SPP modes is weaker due to the fact that SPP modes are predominantly TM polarized; dipoles in the polymer lie in the plane of the layer and are thus poorly matched to the SPP field. For the MEH-PPV based top emitters, Ag proves the most efficient cathode material radiating $24.1 \%$. The values for $\mathrm{Al}$ and $\mathrm{Ca}$ are $9.4 \%$ and $20.9 \%$, respectively.

Turning our attention to the comparison substrateemitting structures, we find that, as expected, although a large percentage of the dissipated power enters the thick silica substrate, only part of this power results in radiation that emerges from the substrate. ${ }^{12}$ For a silver cathode we found the efficiency to be $20.3 \%$, Fig. 3. There is a significant amount of power coupled to the SPP mode associated with the silver cathode, peaking when the emitter-cathode distance is about $30 \mathrm{~nm}$. As this distance becomes much smaller the fraction of power coupled to the SPP mode falls sharply as short range quenching effects take over. ${ }^{13}$ Similar calculations were performed for structures with $\mathrm{Al}$ and $\mathrm{Ca}$ cathodes, their efficiencies being $22.5 \%$ and $15.7 \%$, respectively. For MEH-PPV based substrate-emitting OLEDs, we AIP license or copyright; see http://apl.aip.org/apl/copyright.jsp 
find the outcoupling efficiency to be $30.2 \%, 27.0 \%$, and $18.8 \%$ for the $\mathrm{Al}, \mathrm{Ag}$, and $\mathrm{Ca}$ cathodes, respectively.

Our results show that the choice of cathode material has important consequences for outcoupling efficiency. The best choice of cathode material for any given structure represents a compromise between absorption, power lost to SPP modes, and cavity effects arising from its reflectivity. Aluminum seems to be the best choice of cathode material for substrateemitting structures, whereas Ag proves more efficient for top emitters.

We have shown that, with a suitable choice of cathode material, top-emitting OLED structures may have outcoupling efficiencies comparable with their substrate-emitting counterparts. Further calculations (not shown) indicate that by optimizing the thickness of the organic layer, both structures can achieve somewhat higher outcoupling efficiencies; optimization through layer geometry will be the subject of further study. Still higher efficiencies might be expected if methods of recovering the various guided modes were to be implemented. ${ }^{14}$ Finally, we note that the most efficient devices are likely to be a compromise between electrical and optical performance.
The authors would like to thank both the EPSRC (through the DTI-LINK Project XTRAOLED) and CDT Ltd. for financial support.

${ }^{1}$ V. Bulovic, G. Gu, P. E. Burrows, M. E. Thompson, and S. R. Forrest, Nature (London) 380, 29 (1996).

${ }^{2}$ M.-H. Lu, M. S. Weaver, T. X. Zhou, M. Rothman, R. C. Kwong, M. Hack, and J. J. Brown, Appl. Phys. Lett. 81, 3921 (2002).

${ }^{3}$ H. Riel, S. Karg, T. Beierlein, B. Ruhstaller, and W. Reiss, Appl. Phys. Lett. 82, 466 (2000)

${ }^{4}$ H. Benisty, H. De Neve, and C. Weisbuch, IEEE J. Quantum Electron. 34, 1632 (1998)

${ }^{5}$ P. A. Hobson, S. Wedge, J. A. E. Wasey, I. Sage, and W. L. Barnes, Adv. Mater. (Weinheim, Ger.) 14, 1393 (2002).

${ }^{6}$ H. Raether, Surface Plasmons (Springer, Berlin, 1988).

${ }^{7}$ J. A. E. Wasey and W. L. Barnes, J. Mod. Opt. 47, 725 (2000).

${ }^{8}$ C. W. Tang, S. A. VanSlyke, and C. H. Chen, J. Appl. Phys. 65, 3610 (1989).

${ }^{9}$ H. Becker, S. E. Burns, and R. H. Friend, Phys. Rev. B 56, 1893 (1997).

${ }^{10}$ P. A. Hobson, J. A. E. Wasey, I. Sage, and W. L. Barnes, IEEE J. Sel. Top. Quantum Electron. 8, 378 (2002).

${ }^{11}$ C. M. Ramsdale and N. C. Greenham, J. Phys. D 36, L29-L34 (2003).

${ }^{12}$ M.-H. Lu and J. C. Sturm, J. Appl. Phys. 91, 595 (2002).

${ }^{13}$ I. Pockrand, A. Brilliante, and D. Mobius, Chem. Phys. Lett. 69, 499 (1980).

${ }^{14}$ J. M. Lupton, B. J. Matterson, I. D. W. Samuel, M. J. Jory, and W. L. Barnes, Appl. Phys. Lett. 77, 3340 (2000). 